\title{
Genesis
}

Manuscrits - Recherche - Invention

$50 \mid 2020$

Aragon

\section{Esa Hartmann et Patrick Hersant (dir.), Au Miroir de la traduction. Avant-texte, intratexte, paratexte, Paris, Éditions des archives contemporaines, coll. «Multilinguisme, traduction, création », 2019, $228 \mathrm{p}$.}

Maryvonne Boisseau

\section{CpenEdition}

\section{Journals}

Édition électronique

URL : https://journals.openedition.org/genesis/4976

DOI : 10.4000/genesis.4976

ISSN : 2268-1590

Éditeur :

Presses universitaires de Paris Sorbonne (PUPS), Société internationale de génétique artistique littéraire et scientifique (SIGALES)

\section{Édition imprimée}

Date de publication : 15 juillet 2020

Pagination : 177-183

ISBN : 979-10-231-0679-4

ISSN : 1167-5101

Référence électronique

Maryvonne Boisseau, « Esa Hartmann et Patrick Hersant (dir.), Au Miroir de la traduction. Avant-texte, intratexte, paratexte, Paris, Éditions des archives contemporaines, coll. « Multilinguisme, traduction, création », 2019, 228 p. », Genesis [En ligne], 50 | 2020, mis en ligne le 24 août 2020, consulté le 03 septembre 2021. URL : http://journals.openedition.org/genesis/4976 ; DOI : https://doi.org/10.4000/ genesis.4976 


\section{Comptes rendus d'ouvrages}

Esa Hartmann et Patrick Hersant (dir.), Au Miroir de la traduction. Avant-texte, intratexte, paratexte, Paris, Éditions des archives contemporaines, coll. «Multilinguisme, traduction, création », 2019, 228 p.*

\section{Compte rendu par Maryvonne Boisseau**}

Depuis les années 1970, la réflexion sur la traduction s'est doucement constituée en discipline, la traductologie. Ses orientations dominantes ont fluctué selon les époques et leurs figures de proue, se partageant entre l'attention aux textes et au traduire, et l'attention au traducteur et à la chaîne de production. Récemment, s'agissant de la traduction littéraire, plusieurs publications collectives $^{1}$ ont mis l'accent sur le rapport à la création, pour faire suite à la revendication d'autorité des traducteurs, trop souvent «invisibles », et parce que l'un des objectifs tant de la linguistique contrastive que de la littérature (plus spécifiquement comparée) est de comprendre, par l'examen des différences, comment s'énonce un texte nouveau à partir d'un autre écrit dans une langue étrangère, le passage d'une langue à une autre, d'un texte à un autre, multipliant les possibilités herméneutiques. L'ouvrage Au miroir de la traduction. Avanttexte, intratexte, paratexte, s'inscrit dans cette actualité de la recherche littéraire et traductologique. Par ailleurs, comme l'annoncent les termes de la seconde partie du titre, il se réclame d'un autre paradigme de recherche, celui de la génétique textuelle, ou critique génétique. Également constituée en discipline autonome parallèlement à la traductologie, ayant élargi son champ d'investigation à pratiquement tous les arts impliquant un processus de création, la critique génétique ne pouvait manquer de regarder du côté de la fabrique de la traduction. C'est cette affiliation théorique que, dans leur introduction, Esa Hartmann et Patrick Hersant, mettent en avant, s'inscrivant là aussi dans une lignée d'ouvrages récents dont la parution s'échelonne entre 2011 et 2015 , avec en particulier un numéro de Genesis en 2014, sous la direction de Fabienne Durand-Bogaert ${ }^{2}$. Cette dernière soulignait alors la rareté des études génétiques ayant pour objet la traduction : «Les conditions dans lesquelles une traduction prend forme n'ont guère encore été étudiées : l'atelier du traducteur reste un mystère, comme s'il s'y fomentait quelque entreprise dont seul le résultat - la traduction publiée - importait, le processus effaçant ses traces à mesure ${ }^{3}$ ». Les «traces » en question constituent les éléments très «concrets » d'un dossier génétique et manifestent la réalité complexe d'un écheveau qu'on ne peut démêler qu'en forgeant les outils adéquats. Les auteurs de l'ouvrage empruntent à la génétique textuelle une terminologie (par ex. «avant-texte»), une méthode (dans une certaine mesure), et une ambition, celle de rendre compte de la version éditoriale à partir non seulement du cheminement non linéaire de son élaboration, mais aussi de l'interaction entre les acteurs impliqués dans le processus. La traduction - la version publiée - devient le miroir de l'original tandis que le processus qui l'a engendrée se reflète en elle. Cette métaphore du miroir, qui donne son titre au volume, reviendra sous la plume de plusieurs collaborateurs; tous inscrivent leur contribution à la fois dans le champ de la littérature et de l'herméneutique, et/ou dans celui de la génétique textuelle, manifestant à des degrés divers et de manière plus ou moins équilibrée une orientation vers l'une ou l'autre de ces disciplines. L'ouvrage est organisé en trois parties, "avant-texte», «intratexte» et «paratexte», correspondant à différents états de l'élaboration d'une traduction, incluant l'autotraduction et la création littéraire à partir de la traduction. Les auteurs s'intéressent aux pratiques des acteurs concernés par la traduction, au type de démarches traductives, enfin aux textes eux-mêmes et à leur "paratexte». Non seulement les trois grands genres littéraires qui constituent le champ littéraire sont représentés, mais l'empan historique va de Dante à des auteurs contemporains comme Andréas Becker en passant par Saint-John Perse, Giuseppe Ungaretti ou Jules Verne.

Dans l'introduction, Hartmann et Hersant, se réclamant de Barbara Folkart et d'Henri Meschonnic, soulignent, qu'à l'instar de la poésie, la traduction est un faire, une pæisis. C'est à cet égard qu'elle «offre un objet d'étude privilégié à la critique génétique» qui «entend étudier la genèse d'une œuvre à travers le processus scriptural qui lui a donné naissance » (p. 2). Augmentant l'extension du terme genèse, ils incluent dans le processus non seulement «les documents de travail», mais également les textes qui l'éclairent (toutes formes du discours du traducteur et/ou de l'éditeur). Ils présentent enfin les espaces textuels explorés : l'avant-texte, terrain privilégié de la critique génétique,

(*) En ligne sur le site archivescontemporaines. com

(**) Université de Strasbourg.

1. Citons M. Hennard de la Rochère et I. Weber Henking (dir.), La traduction comme création/ Translation and creativity, Lausanne, Centre de traduction littéraire, coll. «Théorie », 2017 ; L. Belingard, M. Boisseau et M. Sanconie (dir.), La traduction littéraire comme création, Meta, vol. $62, n^{\circ} 3,2017$; C. Berthin, L. Sansonnetti et E. Eels (dir.), Auteurs-traducteurs : l'entredeux de l'écriture, Nanterre, Presses universitaires de Paris Nanterre, 2018.

2. Voir Olga Anokhina, "Cercle, spirale, chaos : cas limites de l'autotraduction », dans l'ouvrage étudié, notamment p. 101 , note 29 , pour quelques références.

3. Genesis, n’ 38, «Traduire», 2014, p. 8. 
constitué de tous les documents qui précèdent la version «finale »; l'intratexte, qui concerne ici l'autotraduction et ses avatars, riche terrain pour une approche génétique; le paratexte, espace périphérique, où se construit notamment le discours réflexif du traducteur. La conclusion de l'introduction, dans une formule sans appel, «il n'est pas de traduction sans traductologie» (p. 8), semble alors englober la critique génétique dans la traductologie, ce qui amène le lecteur à se poser la question du périmètre des disciplines et/ou de leur éventuelle subordination. Il faudra attendre l'article d'Olga Anokhina (deuxième partie) pour que soient mis au premier plan, et nommés, des éléments de méthode de critique génétique, notamment la distinction des différentes phases du processus telles qu'elles ont été définies par Pierre-Marc de Biasi ${ }^{4}$ : pré-rédactionnelle, rédactionnelle, prééditoriale, éditoriale, étapes qui découpent l'avant-texte en séquences observables.

C'est par l'exemple que le lecteur entre de plain-pied dans le vif du sujet avec le premier article signé de Viviana Agostini-Ouafi, «Critique génétique et doute herméneutique. Réflexions de Pézard, traducteur de Dante». L'auteure y commente le texte d'une conférence d'André Pézard, traducteur des œuvres complètes de Dante publiées dans la Bibliothèque de la Pléiade en 1965 pour la première édition, 1983 pour la dernière. Le critique est là face à un matériau de premier ordre : d'abord les différents états du travail (brouillons, mises au net, corrections), ensuite les commentaires du traducteur sur ses propres brouillons (ses hésitations et remords de traducteur), enfin la verbalisation à haute voix du non-dit sous-jacent à ce qui a été écrit (la conférence en question). Il s'agit donc d'une énonciation épilinguistique (épi-génétique ?) et métalinguistique qui se déploie en plusieurs strates inscrites dans le temps long de la traduction proprement réflexive. Les difficultés de traduction sont le lieu de cet examen introspectif verbalisé à la fois à destination de soi-même comme lecteur-interprète-traducteur et d'un lecteur potentiel non naïf. L'article est une introduction à ce cheminement à travers la présentation des explications données par Pézard sur la difficulté d'interpréter, et donc de traduire, un passage ambigu, bien connu des nombreux commentateurs et traducteurs de Dante. C'est un article sur le «labeur» du traducteur : en mettant en lumière «sa [celle de Pézard] méthode de traduction », Agostini-Ouafi travaille à la fois sur les commentaires du traducteur et les variantes de la traduction, mais aussi sur le texte de Dante. Elle démontre ainsi que le matériau d'une critique génétique ne peut être séparé des textes qui en sont à l'origine (original et différentes versions produites), ni des réflexions, lectures et critiques qui accompagnent le travail du traducteur. Les textes étudiés n'appartiennent pas tous à l'avant-texte dans la mesure où nombre de commentaires ont été écrits après coup, corrigés, développés. Ils constituent cependant une documentation complète établie par un traducteur philologue, éminemment conscient de l'importance d'un travail qui procède par approximations successives sans jamais aboutir à LA version définitive.

C'est aussi la place cruciale de l'original que dans "Genèse d'une traduction collaborative : Winds de Hugh Chisholm et Saint-John Perse », Esa Hartmann met en valeur. L'examen des suggestions faites par Saint-John Perse pour amender une version de la traduction du poème Vents proposée par Hugh Chisholm met en lumière le rôle explicatif de ces propositions. Hartmann considère que ce travail, que l'on peut percevoir au premier abord comme une étape de correction et de révision, est en réalité une pratique collaborative entre Saint-John Perse et son traducteur qui, par son ampleur, met le poète en position d'auto-traducteur ${ }^{5}$. Ses annotations mettent deux choses en évidence : $i$ ) la difficulté pour le traducteur d'interpréter la langue du poète; ii) la mainmise du poète sur «son» texte et sa traduction en anglais, les deux formant un tout aux yeux du poète. En ce sens, le dialogue entre le poète et son traducteur appartient à la «genèse » de la traduction. Il renvoie aussi au processus de création du texte premier car Saint-John
Perse, en élucidant les difficultés, révèle une stratégie d'écriture faite de recours aux dictionnaires et à l'étymologie des mots pour choisir le vocable le plus poétique à ses yeux. Toutefois, la mise en lumière de cette étape occulte la genèse de la version élaborée par Hugh Chisholm (qui s'était engagé à en soumettre « trois brouillons » ${ }^{6}$ ), soumise à «mise au point» et «révision finale» (p. 25). L'objectif de l'article n'est pas tant le décryptage de l'activité de langage à travers les différents moments d'écriture-lecture ou lecture-écriture et leur symbolisation marquée notamment par la couleur de l'encre utilisée ou la disposition des variantes, que l'interprétation du poème telle qu'elle peut être guidée par la confrontation de deux écritures : «Notre étude explorera les différents aspects de cette poétique de Vents/Winds, qui se font jour, au sein du processus traductif, à travers la confrontation dialogique des deux écritures traductives à l'œuvre sur le manuscrit [...]» (p. 27). À certains égards, on retrouve dans cette contribution une hésitation qui s'est fait jour dans l'introduction entre traductologie et critique génétique, critique génétique et littérature.

Plus ciblé encore sur l'activité de révision, l'article de Patrick Hersant, «La troisième main : réviser la traduction littéraire», se penche sur cette étape prééditoriale du processus de traduction. Mal définie, elle est tributaire à la fois de la qualité de lecteur du réviseur, de sa maîtrise de la langue cible et de l'idée qu'il s'en fait, des indications qu'il reçoit de l'éditeur, de sa connaissance de l'original. Et si, avec le développement des outils numériques, ce travail est devenu l'un des «métiers » de la traduction, il demeure un travail de l'ombre, moins visible encore que celui

4. Pierre-Marc de Biasi, Génétique des textes, CNRS Éditions, coll. «Biblis», 2011.

5. Suivant en cela Henriette Levillain, auteure d'un ouvrage sur les liens de la création et de la traduction chez Saint-John Perse, Sur deux versants. La création chez Saint-John Perse. D'après les versions anglaises de son œuvre, Paris, Librairie José Corti, 1987.

6. Voir H. Levillain, op. cit., p. 197. 
du traducteur. Avant-dernier maillon d'une chaîne de lecture qui va du traducteur au lecteur, il est rare que les interventions du réviseur sur les épreuves d'une traduction laissent des traces. De plus, lorsque dans le meilleur des cas elles font l'objet d'échanges avec le traducteur, elles peuvent aller contre un «projet traductif» et être à l'origine de relations conflictuelles. Hersant présente cinq cas d'auteurs pour lesquels on dispose de notes et commentaires du réviseur (Saint-John Perse-Eliot-Larbaud, Montale-Reed-Shankland, D'AnnunzioHérelle-Ganderax, Kaplan-Bardos-Cazade, Joyce-Morel-Larbaud), puis il examine les modalités de la révision à partir des traces des échanges entre les différents maillons de la chaîne de lecture. Il s'attache à la forme des observations, aux objectifs du réviseur, aux relations difficiles entre traducteur et réviseur, particulièrement quand l'auteur de l'ouvrage traduit en est lui-même le réviseur. Il s'avère en tout cas malaisé de dresser un portrait du réviseur, et surtout de prendre la mesure de sa contribution au processus traductif et à l'interprétation du texte qui peut en résulter en raison de la rareté des traces exploitables. Il n'en reste pas moins que, le plus souvent, le sens des révisions est d'aller vers un lissage des aspérités d'une traduction, au risque d'un «aplatissement» de la traduction, même si certains réviseurs s'en défendent.

Il est cependant des collaborations heureuses comme le raconte Julia Holter dans un article quasi autobiographique, «Résonance "traductrice", ou traduire Anna Glazova à quatre mains ». Une expérience de traduction poétique complexe est exposée en mêlant plusieurs niveaux de sorte que ce qui relève de la matérialité du processus traductif proprement dit ne se trouve pas véritablement dégagé des questions herméneutiques développées par Holter. Cette dernière, russophone, explique comment le russe d'Anna Glazova l'a surprise par une "étrangeté » qu'elle n'arrivait pas à situer (p. 72). De fait, la langue de Glazova est imprégnée de résonances de l'allemand du poète Paul Celan, mais aussi de Rilke, ce que la traductrice découvre grâce à son collaborateur, JeanClaude Pinson qui, lui, ne connaît pas le russe. À cette situation interlingue, se superpose celle de Holter dont la propre langue se trouve en quelque sorte reléguée à l'arrière-plan de sa vie puisqu' elle dit travailler maintenant en anglais américain et en français, sans nécessairement en connaître toutes les subtilités. Il s'agit donc d'une collaboration où les rôles sont distribués selon les connaissances linguistiques de l'un et l'autre. Et c'est ainsi le récit de cette quête du sens du poème, dialogue émaillé d'allusions philosophiques suscitées par le ton du poème et par les échos explicites qu'il envoie, que cette contribution fait entendre. Le risque d'éparpillement est là cependant, avec l'impression qu'à la faveur des allusions philosophiques, l'on «picore», dans les philosophies anciennes (la Grèce), ou plus proches de notre temps (allemande, française, italienne), les concepts supposés donner les clés de son interprétation. Le lecteur devra alors faire la part de ce qui appartient à la genèse de la traduction et/ou à la genèse de l'interprétation, et prendre conscience de l'éclectisme philosophique des allusions repérées. Cette expérience de collaboration (Holter-Pinson) permet de vérifier que les éclairages mutuels démultiplient les possibilités de compréhension et de rédaction; son récit introduit cependant une subjectivité de nature à dévoyer une démarche scientifique de critique génétique visant, à partir de traces matérielles codées, à une certaine objectivité des analyses et des conclusions qu'on en tire, quand bien même la subjectivité serait inhérente à l'activité de traduction.

L'intérêt de l'article suivant, d'Emilio Sciarrino, «Giuseppe Ungaretti et l'épreuve de la traduction ${ }^{7}$ », tient au fait qu'il rassemble différents aspects abordés dans les articles précédents : travail collaboratif, conception hiérarchique de la collaboration, interventions de révision plus ou moins tatillonnes et, surtout, le rôle crucial de la traduction dans la création littéraire ainsi que l'intérêt d'une approche génétique. L'auteur rappelle que toute l'œuvre d'Ungaretti est «caractérisée par un incessant mouvement de traduction, d'auto-traduction et de retraduction» (p. 81), fait rarement mentionné, d'où l'intérêt de l'étude de ses archives qui «fait apparaître la dimension réellement bilingue de son écriture, où la traduction joue le rôle de moteur latent mais décisif » (p. 81). À titre d'illustration, Sciarrino décrit le voyage vers la traduction d'un poème très personnel d'Ungaretti : traduction par un traducteur belge, interventions du poète, autres traductions confiées à d'autres traducteurs, interventions du poète, modifications de l'original sous l'influence de la version française. L'article mentionne une dernière traduction effectuée par Philippe Jacottet, sans collaboration semble-t-il, et publiée en 1970 (année de la mort d'Ungaretti). Ce processus collaboratif et cette dynamique d'écriture, que Sciarrino décrit comme «un processus d'écriture bilingue circulaire» (p. 93), sont plus ou moins bien assumés par le poète qui a de la traduction une vision hiérarchique. Force est de constater cependant qu'Ungaretti a modifié considérablement la première version du poème en question à plusieurs reprises sous l'influence de la traduction, jusqu'à "atteindre un état d'équilibre définitif» (p. 93). L'activité de traducteur d'Ungaretti dans sa relation à la création poétique est primordiale : «L'importance de la traduction chez cet auteur confirme du reste que son écriture s'enracine dans un bilinguisme profond. Ainsi même une traduction apparemment insatisfaisante peut en réalité jouer un rôle crucial, et constituer une étape essentielle dans le cheminement de l'écriture vers la version définitive» (p. 93).

Si cette première partie explore différentes situations qui toutes, de manière plus ou moins lâche, sont des situations de collaboration, la deuxième s'en éloigne pour envisager la traduction dans sa dimension de dédoublement d'une langue à l'autre par

7. Sciarrino indique (note 5, p. 81) que son article a fait l'objet d'une première publication contenant la reproduction intégrale des documents discutés : «Giuseppe Ungaretti et le processus de création circulaire. La genèse de Gridasti : soffoco », Genesis, n 46, «Entre les langues », 2018, p. 145-158. 
un auteur-traducteur. Il existe cependant des «cas frontaliers» qu' aborde Olga Anokhina dans l'article qui ouvre cette section : «Cercle, spirale, chaos : cas limites de l'autotraduction». Entre la traduction collaborative et l'autotraduction, il existe, écrit-elle, des pratiques intermédiaires (semi-autotraduction, autotraduction partagée, auto-traduction auctoriale, autotraduction indirecte). Par ailleurs, elle distingue une «écriture simultanée » d'une «écriture consécutive» (cas «canonique» de l'autotraduction). Prenant en compte les travaux sur l'autotraduction faits dans le cadre de la traductologie, Anokhina interroge alors «la pertinence des critères ayant présidé à l'élaboration [des typologies des pratiques autotraductives]» (p. 99). Les cas observés (Heinrich Heine, Kafka traduit par Borges, Celan) l'amènent à écarter le critère paratextuel en raison de son opacité pour lui substituer le critère avant-textuel. Elle représente ensuite par différentes figures (cercle, spirale en deux dimensions, spirale en trois dimensions et mouvement chaotique) le fonctionnement des écritures simultanée et consécutive, selon les mouvements d'un texte vers l'autre, dans un sens ou/et dans l'autre, selon aussi le temps qui sépare ou non les deux productions. L'étude des documents de travail d'écrivains plurilingues montre que «l'écriture simultanée ne relève pas de l'autotraduction à proprement parler : elle constitue une stratégie créative à part» (p. 104), ce qui n'empêche pas une publication séparée dans une version monolingue. L'argumentation de l'auteure aboutit à établir un distinguo net entre l'autotraduction au sens habituel du terme et «l'écriture plurilingue simultanée » : la première intervient au moment de l'étape pré-éditoriale, la seconde au moment de la rédaction. Anokhina nuance toutefois cette classification en expliquant que l'autotraduction, chez certains auteurs, sert à corriger un texte en cours d'élaboration. Son statut n'est donc pas le même et cette autotraduction, miroir du texte initial, n'est pas destinée à être publiée. Cet article qui fonde son argumentation sur un examen du matériau avant-textuel ébranle les typologies de l'autotraduction établies par les traductologues. On peut voir dans les différentes pratiques un continuum, mais du point de vue de la genèse, il semble bien qu'on ait affaire à deux modes d'écriture différents.

Sara De Balsi réfute, elle aussi, le critère paratextuel (ici le discours de l'écrivain) utilisé pour justifier l'orientation d'une œuvre. Sa contribution, «Crise du monolinguisme. Les poèmes hongrois d'Agota Kristof et leurs autotraductions », met à mal l'opinion communément admise, sur la foi des déclarations de l'auteure, que l'œuvre d'Agota Kristof est «rigoureusement monolingue» (p. 112). La publication posthume des poèmes de jeunesse, écrits en hongrois et traduits en français, révèle qu' au contraire une circulation a bien eu lieu entre les poèmes écrits en hongrois et la production en langue française. Selon l' auteure, l'ambiguité entretenue par Kristof sur son activité d'autotraduction proviendrait de la conception qu'elle avait de la création littéraire à laquelle elle accordait plus d'importance qu'à la langue dans laquelle elle se réalisait. Ce déni affectif envers une langue, quelle qu'elle soit, l'amène à sacraliser l'écriture et la réécriture en masquant le geste autotraductif et son importance dans la création.

Autre genre littéraire, autre langue : «Au-delà du voile de Slimane Benaïssa: une autotraduction oubliée?», la même problématique d'une autotraduction invisible est décrite par Chiara Lusetti du point de vue de la littérature comparée. L'interrogation du titre suscite immédiatement une réaction de la part du lecteur: oubliée par qui ? l'auteur? le critique? le spectateur de la pièce? Le choix du mot oubliée ne semble pas adéquat à la situation décrite. La différence entre une première version en arabe de la pièce et sa version française peut en effet s'expliquer par la rupture de l'auteur avec son pays et l'écart entre deux publics qui envisagent, pour des raisons historiques et sociologiques, le contexte de la pièce de théâtre de manière radicalement différente. La question que l'on pourrait poser, en revanche, n'est pas tant celle de l'autotraduction que celle des critères présidant à une auto-adaptation de la pièce intégrant un traitement des références culturelles par l'auteur. Ces transpositions d'une version à l'autre ont-elles laissé des traces dans des brouillons? La version en arabe algérien a été «recréée » à plusieurs reprises lors des représentations mais sa version écrite est restée inédite. Doit-on considérer alors ces versions jouées en arabe comme les «brouillons» de la version en français?

L'étude littéraire qui suit est consacrée à l'écrivain franco-américain Raymond Federman (1928-2009) : «Raymond Federman : écrivain bilingue, autotraducteur, traductologue?» L'auteure, Amanda Murphy, dit s'inscrire dans une perspective génétique qui «permet d'interroger la clôture du texte qu'induit la publication » (p. 133) et qui incite à remettre en question la version publiée. Cependant, le titre de cette contribution met l'accent sur l'auteur lui-même plus que sur ses textes. Federman, écrivain juif parisien, sauvé de justesse d'une rafle en 1942, émigrera aux États-Unis en 1947 et cherchera à se libérer du traumatisme causé par cet événement par la création. Il écrira alors, simultanément en anglais et en français, les deux versions étant proposées parallèlement lors de la publication, mais dans une présentation graphique différente. On retrouve en partie la problématique théorisée par Anokhina, illustrée par un chevauchement des langues d'écriture. L'étude des archives de Federman montre qu'avant la publication de ses textes, de nombreuses autres versions ont été écrites, dans les deux langues mêlées. Lui-même le vivait ainsi : «[...] pour moi, le français et l'anglais semblent toujours se chevaucher, semblent vouloir fusionner, vouloir se rassembler, vouloir s'embrasser, s'entendre» (p. 136). Se pose alors la question du passage de la langue de départ à la langue d'arrivée alors que se construit une langue, celle de l'écrivain qui, s'il fallait donner une réponse à l'interrogation du titre de l'article, n'est ni traductologue, ni autotraducteur, mais bel et bien un écrivain dont le mode de création semble ancré dans l'indivision (au sens 
juridique du terme) de deux langues qui, travaillées ensemble, projettent dans le miroir l'image d'un mouvement chaotique (Anokhina, p. 104). Ce chaos aboutit à la création de textes uniques, préludes à la version publiée, ce qu'une étude génétique, au ras des avant-textes, devrait pouvoir démêler sans préjuger de leur interprétation.

La pratique de la traduction irrigue la création littéraire, comme le montre aussi la contribution de Fernand Salzmann, «L'infraction par la traduction : Le Fou d'Elsa d'Aragon ». Le poème est une «réécriture de la fable du Medjnoûn Leylâ, l'une des histoires les plus connues dans la tradition littéraire arabo-persane» (p. 145). Placée sous le signe de «l'infraction», la traduction pose la question de l'utilisation d'une langue «fautive», par laquelle un langage se réinvente et grâce à laquelle «se traduit une irréductible altérité» (p. 146). L'analyse de deux exemples extraits de l'œuvre, «L'encore», dernier poème de la première partie, qui joue sur le russe et le français, et l'impossible traduction du prénom «Elsa » en arabe, permet à l'auteur de montrer que le poète refuse «la domestication du texte étranger» et toute hiérarchisation des langues. À mi-chemin entre la traductologie et la littérature, l'article opère des va-et-vient entre le texte et les commentaires du poète, inscrits dans le poème, pour redire l'importance de la traduction en tant que «ressource» au service d'un renouvellement de la poésie, ce qu'au fond tous les poètes ambitionnent plus ou moins secrètement de faire.

Fermant cette deuxième partie de l'ouvrage, la contribution de Mathilde Vischer «L'œuvre d'Andréas Becker, ou la langue "traduite" », nous ramène, par le biais d'une étude traductologique, sur les rivages de la génétique textuelle. L'auteure, s'inspirant de Arno Renken ${ }^{8}$, part de l'idée que la traduction est un «geste de différenciation» capable «d'inquiéter nos ordres » (p. 157) pour «montrer en quoi les textes de Becker peuvent être perçus comme une mise en abyme de ce que fait la traduction» (p. 156). À partir d'une expérience linguistique singulière, celle d'un auteur qui écrit dans une langue (le français) qui n'est pas «sa » langue, Vischer offre ici une réflexion sensible sur ce que la traduction fait, et «fait à la langue et au texte original» (p. 162). Elle illustre aussi ce qu'une réflexion sur la complexité déstabilisante des relations entre des textes, qui ne sont ni des originaux ni des traductions, permet de saisir du processus traductif (perspective génétique). Le traducteur, cet écrivain de l'étrangeté, est nécessairement inscrit dans ce processus (perspective traductologique) que l'étrangéisation calculée d'une langue (au sens culiolien de «calcul du sens ») met en lumière. Jouant sur les deux sens du mot traduction, processus et production, Vischer n'oublie pas que les textes s'adressent à un lecteur dont «le rapport au monde et à la langue » est mis à l'épreuve par la vulnérabilité des textes et des langues que l'accès au processus de création révèle.

L'autotraduction manifeste un trouble dans l'énonciation et révèle que l'instance énonciative n'est jamais unique mais faite de voix multiples qui se mêlent dans la construction du texte et le processus traductif où se conjuguent interprétation simultanée et différée, traduction privilégiée des émotions dans une langue ou dans l'autre, écriture parallèle ou alternée. Elle rend les notions d'original et de version traduite caduques, met en question la notion de trahison, défie le monolinguisme et, dans le cas d'une «écriture plurilingue simultanée», déstabilise la lecture du texte. Elle est elle-même un défi pour la traduction et, lorsque les archives existent, une mine pour la critique génétique.

L'importance du paratexte (dernière partie) n'a pas échappé aux traductologues ${ }^{9}$. La critique génétique s'en saisit maintenant pour pallier l'absence de brouillons dans bien des cas. Ezra Pound, par exemple, pour lequel l'enjeu de la traduction était l'invention de la langue, comme le décrit Irena Kristeva dans l'article «Ezra Pound : la traduction rénovatrice», a clarifié sa pratique de la traduction dans un essai, Guido's Relations, et dans les notes qui accompagnent sa propre traduction des sonnets de Cavalcanti. L'auteure vérifie la pertinence des propos de Pound en analysant la traduction d'un sonnet de Cavalcanti et la transcréation d'un poème de Li Po. Convoquant Yves Bonnefoy, elle rappelle l'importance des rythmes et de «l'état chantant de la langue» auxquels Pound, musicien lui-même, était particulièrement sensible. Les concepts de logopæia (« usage contextuel des connotations actualisées par les jeux linguistiques»), phanopœia («projection des images sur l'imagination visuelle») et melopæia («la qualité musicale qui dirige le sens »), trois traits distinctifs de la poésie selon Pound, sont mis à contribution pour caractériser les différentes œuvres : les Cantos pour le premier, les traductions du poète chinois Li Po pour le deuxième, les traductions des Sonnets de Cavalcanti pour le troisième. Dans tous les cas, la traduction ne saurait être transparente. Elle est «transcréation» et c'est à ce prix qu'elle renouvelle la langue.

Dans un autre ordre d'idée, Marian Panchón Hidalgo, dans «La paratraduction des œuvres politiques surréalistes sous le franquisme (1959-1975)», s'appuie sur la notion de «paratraduction», définie par le traductologue espagnol José Yuste Frías, pour examiner la façon dont les traductions des poètes surréalistes français (en l'occurrence Aragon et Breton) et l'ouvrage collectif Position politique du surréalisme ont été présentés par l'édition espagnole au temps de la censure franquiste de la période allant de 1959 à 1975 . Cette notion renvoie «à l'ensemble des productions verbales, iconiques, verbo-iconiques et matérielles des paratextes figurant dans

8. Philosophe et comparatiste, Arno Renken est l'auteur de Babel heureuse. Pour lire la traduction, Paris, Van Dieren Éditeur, coll. «Par Ailleurs Riponne», 2012.

9. Des publications récentes en témoignent, par exemple : H. Jansen et A. Wegener (éd.), Authorial and Editorial Voices in Translation, t. I et II, Montréal, Éditions québécoises de l'œuvre, coll. «Vita traductiva », 2013; I. Génin et J. Stephens (dir.), Quand les traducteurs prennent la parole: préfaces et paratextes traductifs, Palimpsestes, $\mathrm{n}^{\circ} 31$, Presses Sorbonne Nouvelle, 2018. 
les traductions [...]» (p. 179) et incluent les «dossiers et rapports de censure» (p. 181), «péritexte» et «épitexte» dans la terminologie de Genette. En somme, ces textes renseignent davantage le lecteur sur l'histoire de la réception des traductions et sur les raisons de leur publication que sur les traductions elles-mêmes. Ils ont une portée idéologique, sinon politique, et montrent comment les éditeurs contournent la censure ou la servent, conduites éditoriales ambiguës tributaires des opinions politiques des éditeurs eux-mêmes qui seront diversement décryptées selon les lecteurs.

Quand le paratexte va à l'encontre des opinions reçues... Dans «Amédée Pichot, traducteur traductologue », Frédéric Weinman s'attache à redorer la réputation d'un traducteur tenu en piètre estime par ses pairs. Amédée Pichot, traducteur de grands auteurs anglais du XIX ${ }^{\mathrm{e}}$ siècle (et donc «passeur» clairvoyant), a été sévèrement critiqué au $\mathrm{xx}^{\mathrm{e}}$ siècle par des traducteurs reconnus, tels Robert Escarpit et Sylvère Monod. Or, les paratextes de Pichot, lus avec un recul suffisant, proposent une réflexion qui fait de lui, aux yeux de Weinman, et au risque d'un anachronisme, un traductologue. Les réflexions de Pichot témoignent d'une conscience scrupuleuse à l'égard du texte original. Que ce soit en traduisant de la poésie en prose, ou de la prose en respectant le style et la forme, l'ethos de Pichot est en phase avec certaines conceptions actuelles de la traduction qui préconisent le «respect» du texte et l'adaptation de la méthode de traduction à la particularité de chaque œuvre. Dans le même temps, il s'agit aussi pour le traducteur de respecter la langue littéraire française. Ainsi cet article défend-il la cohérence d'une pensée de la traduction conforme aux attentes de l'époque, de l'idée qu'on se faisait des «belles lettres » et de la relation entre l'écrivain et le traducteur. Ce dernier ne surpasse pas l'auteur et s'efforce, par conséquent, de cultiver l'art du compromis qui voit le «bon usage» l'emporter sur l'audace. C'est peut-être cela qu'un traducteur plus jeune pouvait reprocher à son aîné.
La toute dernière contribution, «Texte ou paratexte. Jules Verne en chinois : une traduction de 1903 » de Florence Xiangun Zhang, met en lumière le rôle d'éducation des traductions. En réaction à la littérature traditionnelle chinoise qui ne favorise pas le développement d'une pensée moderne, des intellectuels comme Liang Qichao (1873-1929) appellent à traduire des romans occidentaux, particulièrement politiques et scientifiques, afin d'éveiller les Chinois à une pensée du progrès. Lu Xun (18811936), considéré comme le plus grand écrivain chinois du $\mathrm{xx}^{\mathrm{e}}$ siècle, traduira alors de nombreuses œuvres occidentales, à partir de leur version en japonais. Il s'intéressera particulièrement à Jules Verne pour promouvoir une culture scientifique et de progrès. L'auteure se penche sur les «paratextes» ajoutés par le traducteur et qui accompagnent la traduction ou s'insèrent sous différentes formes dans le texte traduit (préfaces, intertitres, commentaires). Ces textes exposent la conception de la littérature du traducteur, empruntent à la littérature traditionnelle chinoise certaines contraintes formelles et certains signaux, et proposent en même temps une appréciation du texte traduit. Il s'agit d'acclimater le roman d'anticipation à la culture d'accueil pour «éduquer le lecteur» (p. 209). Le rôle de ces commentaires paratextuels est de «ménager un seuil assez bas pour que l'œuvre reste accessible au plus grand nombre possible de lecteurs» (p. 210).

Si l'on devait établir une typologie des textes qui constituent le paratexte, on distinguerait sans doute ceux qui traitent du processus en ayant une répercussion sur le produit final (échanges, commentaires, notes), ceux qui éclairent les tenants et aboutissants d'un projet traductif (préfaces, postfaces) et ceux qui déterminent la réception des traductions (préfaces éditoriales, avertissements divers, censure). Ces trois catégories pourraient être affinées par des critères tenant compte du lectorat auquel est destinée la traduction, de la situation culturelle et politique de la culture d'accueil, du mode de traduction. Dans tous les cas, le paratexte est destiné à faciliter l'accès aux traductions, tout particulièrement lorsque celles-ci sont éloignées de la culture d'accueil. Toutefois, suffit-il, comme l'ont globalement fait les auteurs de ces quatre contributions, d'analyser ces textes dans une perspective littéraire, traductologique, historique ou culturelle, comme un lecteur «savant» peut le faire ? Ou bien conviendrait-il d'aller chercher dans leur avant-texte, si on peut y accéder, les traces de leur élaboration afin de mettre au jour l'implicite de ces textes publiés, clarifiant ainsi les enjeux non seulement des choix opérés par le traducteur au cours du processus mais aussi ceux de politique éditoriale qui président à la publication des traductions et facilitent leur acculturation?

Ces interrogations ouvrent un double questionnement. Le premier est celui de la fluctuation des frontières entre les disciplines et de la gestion de l'interdisciplinarité. Une histoire épistémologique de la traductologie montrerait comment la discipline s'est sédimentée par période : la période linguistique qui, parfois, a conduit à une approche des textes selon une méthodologie réductrice faisant oublier la théorisation à l'œuvre, le virage culturel inscrivant les études traductologiques dans un vaste mouvement de globalisation, la période historico-culturelle qui s'est récemment attachée à l'histoire des traductions, jusqu'à ce tournant génétique privilégiant, pour l'instant, la traduction littéraire et se nourrissant de littérature comparée. Sans doute ne trouve-t-on pas encore, dans cet ouvrage, l'attention minutieuse aux marqueurs précis que sont, par exemple, les ratures et biffures, la disposition sur la page, les couleurs d'encre, la hiérarchie des variantes, à laquelle la génétique textuelle nous a habitués. Sans doute remarquet-on une ambivalence entre la perspective génétique annoncée et la littérature, qui semble faire pencher la balance vers l'herméneutique. Une hésitation perceptible d'ailleurs entre les termes traductologie et traduction est symptomatique de ce qui peut apparaître comme un tâtonnement. La deuxième grande question est celle du traducteur, façonné comme traducteur par 
le texte qu'il énonce, version après version. En effet, plus que dans la répartition des contributions entre «avant-texte», «intratexte» et «paratexte», la cohérence de l'ouvrage, en dehors du fait qu'il se consacre à la traduction littéraire et à des traducteurs dont les archives sont accessibles, est à chercher, au fil des articles, dans cette présence à facettes. En définitive, il s'agit de délivrer le traducteur de ce face-à-face avec lui-même : "Il faut n'avoir jamais fait l'expérience du traduire pour méconnaître cette donnée fondamentale : c'est à soimême que la traduction renvoie, encore et toujours. Sa visée peut élire l'Autre comme destinataire, mais dans le temps où elle se déroule, l'opération de traduction n'a toujours que soi-même comme point de butée 10 . Il y a quelque chose de l'enquête policière dans cette fouille des archives, une quête de la vérité de l'œuvre. Encore faut-il savoir se laisser surprendre par l'avant-texte. De ce point de vue, cet ouvrage apporte une pierre à la construction d'une critique génétique de la traduction littéraire, dans le cadre d'une tradition traductologique qui, en quelques décennies, a contribué à placer l'activité de traduction, longtemps négligée, sur le devant de la scène de la recherche en sciences humaines et sociales.

Nicolas Donin, Un siècle d'écrits réflexifs sur la composition musicale. Anthologie d'auto-analyses, de Janáček à nos jours, Genève, Droz, HEM (Haute école de musique de Genève), 2019, 717 p.

\section{Compte rendu par Céline Conus*}

Un compositeur peut écrire d'au moins deux manières : de la musique et sur la musique. C'est ce second aspect que mettent ici en jeu les textes de trente compositeurs réunis par Nicolas Donin dans un impressionnant volume rose. La spécificité de ces écrits réside avant tout en leur dimension autoréflexive, car c'est sur leur propre œuvre, plus encore sur leur façon de faire œuvre que s'expriment ici les créateurs. Ces textes ne sont donc pas des traces laissées par une œuvre en train de se faire, mais, proposition audacieuse, des réflexions métagénétiques, soit sur l'œuvre en cours d'écriture, soit quand advient un écart temporel entre l'œuvre et le texte, sur comment l'œuvre a été faite. L'auto-analyse n'étant pas un «genre constitué» (p. 17), les types de témoignages réunis par Donin varient : extrait de journal, texte littéraire, note de travail, article ou encore interview; cette hétérogénéité générique est sans aucun doute un des points forts de l'anthologie. Un autre intérêt évident de ce corpus réside en la rareté des textes qui le constituent - certains documents ont été traduits en français pour l'occasion, d'autres sont inédits. En introduction, Nicolas Donin, qui poursuit par là des travaux en génétique de la création musicale entamés il y a plusieurs années déjà ${ }^{11}$, précise qu'aucun de ces écrits n'a d'ambition théorique. D'une telle ambition, l'anthologie se dégage également : elle ne prétend ni au statut de modèle, ni à établir un «corpus normatif » (p. 18). Le mode de présentation des textes en témoigne d'ailleurs : chacun est brièvement introduit et contextualisé, sans être véritablement analysé. Le lecteur est laissé libre, invité à plonger lui aussi dans ce que les compositeurs disent de l'acte créatif, de sa structure, de sa chronologie, de son importance ou encore de sa valeur en tant que tel. Nicolas Donin semble avoir pensé le volume comme une base de travail, un nouveau genre de matériel mis à la disposition des chercheurs, notamment des doctorants en art. Ajoutons encore que l'ordre chronologique dans lequel sont présentés les documents - qui couvrent un peu plus d'un siècle, de 1897 à 2014 permet de constater l'extrême variété des processus de création musicale ainsi que leur évolution, marquée notamment par l'avènement de l'informatique. Telle une anthologie-laboratoire (ou conservatoire?), l'ouvrage ne s'adresse donc pas uniquement aux musicologues mais est susceptible d'intéresser un lectorat plus large.

Ces documents répondent-ils à la définition des sources sur lesquelles s'appuient les études génétiques? Même si l'écriture introduit nécessairement une distance avec soi-même, on peut craindre en effet que le caractère autoréflexif de ces commentaires floute le «témoignage» qu'ils apportent. Nicolas Donin l'admettait lui-même en 2015 : «Solliciter la parole de l'auteur, pour le généticien, peut n'être qu'une occasion de se piéger soi-même ${ }^{12}$.» Réjouissons-nous au contraire : c'est leur «inobjectivité » qui intéressera précisément. Ces récits trahissent en effet moins une connaissance de la genèse qu'une croyance, et celle-ci accompagne et peut-être guide à sa manière le processus lui-même. À cet égard, la différence entre un témoignage volontaire, «auto-généré », si l'on peut dire, et une interview ou une enquête, par exemple, sollicitée, mériterait d'être prise en considération. La dimension réflexive permet en outre plusieurs niveaux de lecture : il y a ce que dit le compositeur - sa propre histoire de la genèse attestant de ce qui a fait événement pour lui dans son invention - et il y a comment il le dit, sous quelle forme, avec quels mots (la poétique de son autocritique). Quoi qu'il en soit, il s'agit bien d'un geste d'autogenèse - Donin parle d'auto-analyse, qui suppose, selon lui, une forme de déconstruction de l'œuvre. Le musicologue considère en effet l'autogenèse comme une démarche «à rebours » (p. 16); le contraire de la composition en somme, qui est, elle, «synthèse consistant à tenir ensemble des éléments de façon à les intégrer dans un tout» (p. 16). Nous

10. Fabienne Durand-Bogaert, «Traduire : la butée sur soi », Fabula, n 7, «Traduire», Presses universitaires de Lille, 1986, p. 51, cité par Daria Sinichkina, «L'expérience de la Fabrique des Traducteurs : les brouillons de traduction comme espace de confrontation et de réappropriation », Genesis, n $^{\circ} 38,2014$, p. 99 (note 2).

(*) Université de Lausanne.

11. Voir Genesis, n 31, «Composer», 2010, en ligne sur openedition.org, ou Genèses musicales, N. Donin, A. Grésillon et J.-L. Lebrave (dir.), Paris, SUP, 2015 ou encore Nicolas Donin, «La musique, objet génétique non identifié?», Littératures, 2015, $\mathrm{n}^{\circ}$ 78, p. 105-116.

12. Nicolas Donin, «La musique, objet génétique non identifié ?», art. cit., p. 112 